\title{
ON THE FUNCTIONAL-INTEGRAL EQUATION OF VOLTERRA TYPE WITH WEAKLY SINGULAR KERNEL
}

\author{
Aldona Dutkiewicz
}

Communicated by Stevan Pilipović

\begin{abstract}
We give sufficient conditions for the existence of $L^{p}$-solution of a Volterra functional-integral equation in a Banach space. Our assumptions and proofs are expressed in terms of measures of noncompactness.
\end{abstract}

\section{Introduction}

Let $E, F$ be Banach spaces, $D=\left[0, d_{1}\right] \times \cdots \times\left[0, d_{m}\right]$ and

$$
D(t)=\left\{s=\left(s_{1}, \ldots, s_{m}\right) \in R^{m}: 0 \leqslant s_{i} \leqslant t_{i}, \quad i=1, \ldots, m\right\}
$$

for $t=\left(t_{1}, \ldots, t_{m}\right) \in D$. Denote by $L^{p}(D, E)(p>1)$ the space of all strongly measurable functions $x: D \mapsto E$ with $\int_{D}\|x(t)\|^{p} d t<\infty$, provided with the norm $\|x\|_{p}=\left(\int_{D}\|x(t)\|^{p} d t\right)^{1 / p}$.

We consider the following functional-integral equation of Volterra type

$$
x(t)=\phi\left(t, \int_{D(t)} K(t, s) g(s, x(s)) d s\right)
$$

with the kernel $K(t, s)=\frac{A(t, s)}{|t-s|^{r}}, 0<r<n(t, s \in D, t \neq s)$. We give sufficient conditions for the existence of a solution $x \in L^{p}(D, E)$ of $(1)$. Moreover, for $r<1$ we present one-dimensional result involving a generalized Osgood condition. Our considerations are inspirated by a paper of Darwish [5] concerning the functionalintegral equation of Hammerstein type. The existence of $L^{1}$-solution of functionalintegral equation of Hammerstein type was studied in [4] and when $g(s, x)=x$ we get an equation considered in [3]. In [15] Szufla has established the existence of $L^{p}$-solution of Hammerstein integral equation with weakly singular kernel.

Throughout this paper we shall assume that:

2000 Mathematics Subject Classification: 45 N05.

Key words and phrases: Functional-integral equation, $L^{p}$-solutions, measure of noncompactness. 
$1^{\circ}(t, x) \mapsto \phi(t, x)$ is a function from $D \times E$ into $E$ such that

(i) $\phi$ is strongly measurable in $t$ and continuous in $x$;

(ii) $\|\phi(t, x)-\phi(\tau, y)\| \leqslant\left|a_{1}(t)-a_{1}(\tau)\right|+b_{1}\|x-y\|$ for $t, \tau \in D$ and $x, y \in E$, where $a_{1} \in L^{p}(D, R)$ and $b_{1} \geqslant 0$

(iii) $\phi(0,0)=0$

$2^{\circ} A$ is a bounded strongly measurable function from $D \times D$ into the space of continuous linear mappings $F \mapsto E$;

$3^{\circ}(t, x) \mapsto g(t, x)$ is a function from $D \times E$ into $F$ such that

(i) $g$ is strongly measurable in $t$ and continuous in $x$;

(ii) $\|g(t, x)\| \leqslant a_{2}(t)+b_{2}\|x\|$ for $s \in D$ and $x \in E$, where $a_{2} \in L^{p}(D, R)$ and $b_{2} \geqslant 0$.

In what follows we shall need the following lemmas:

LEMma 1. The linear integral operator

$$
(S x)(t)=\int_{D} K(t, s) x(s) d s \quad\left(x \in L^{p}(D, E), t \in D\right)
$$

maps $L^{p}(D, E)$ into itself continuously. Moreover,

$$
\|S\| \leqslant a Q, \text { where } a=\sup \{\|A(t, s)\|: t, s \in D\}
$$

and

$$
\frac{2 \pi^{n / 2}(\operatorname{diam} D)^{n-r}}{(n-r) \Gamma(n / 2)}=Q \geqslant \int_{D} \frac{d s}{|t-s|^{r}} \quad \text { for all } t \in D .
$$

Lemma 2. Put $G(x)(t)=g(t, x(t))$ for $x \in L^{p}(D, E)$ and $t \in D$. Then $G$ is a continuous mapping of $L^{p}(D, E)$ into itself.

For the proofs we refer for example to [15].

Denote by $\alpha$ and $\alpha_{1}$ the Kuratowski measures of noncompactness in $E$ and $L^{1}(D, E)$, respectively. For any set $V$ of functions belonging to $L^{1}(D, E)$ denote by $v$ the function defined by $v(t)=\alpha(V(t))$ for $t \in D$ (under the convention that $\alpha(X)=\infty$ if $X$ is unbounded), where $V(t)=\{x(t): x \in V\}$. The next lemma clarifies the relation between $\alpha$ and $\alpha_{1}$.

Lemma 3. ([7, Th.2.1]; and [16, Th.1]) Assume that $V$ is a countable set of strongly measurable functions $D \mapsto E$ and there exists an integrable function $\mu$ such that $\|x(t)\| \leqslant \mu(t)$ for all $x \in V$ and $t \in D$. Then the corresponding function $v$ is integrable on $D$ and

$$
\alpha\left(\left\{\int_{D} x(t) d t: x \in V\right\}\right) \leqslant 2 \int_{D} v(t) d t .
$$

If, in addition $\lim _{h \rightarrow \infty} \sup _{x \in V} \int_{D}\|x(t+h)-x(t)\| d t=0$, then

$$
\alpha_{1}(V) \leqslant 2 \int_{D} v(t) d t
$$




\section{The main results}

Let $H: D \mapsto R_{+}$be a measurable function such that the function $(t, s) \mapsto$ $\|A(t, s)\| H(s)$ is bounded on $D \times D$.

TheOREM 1 . Let $1^{\circ}-3^{\circ}$ hold and $0<r<n$. If

$$
\alpha(g(s, X)) \leqslant H(s) \alpha(X)
$$

for any $s \in D$ and for any bounded subset $X$ of $E$, then the equation (1) has a solution $x \in L^{p}(D, E)$.

In the case, when $r<1$, we can apply the famous Mydlarczyk theorem [12, Th.3.1], and consequently we obtain a stronger theorem if we replace (3) by the condition (5) given below.

TheOREM 2. Let $\omega: R_{+} \mapsto R_{+}$be a continuous nondecreasing function such that $\omega(0)=0, \omega(t)>0$ for $t>0$ and

$$
\int_{0}^{\delta} \frac{1}{s}\left[\frac{s}{\omega(s)}\right]^{\frac{1}{1-r}} d s=\infty \quad(\delta>0) . \quad(c f .[12])
$$

Let $1^{\circ}-3^{\circ}$ hold, $0<r<1$ and $J=[0, d]$ be a compact interval in $R$. If

$$
\alpha(g(s, X)) \leqslant \omega(\alpha(X))
$$

for any $s \in J$ and for any bounded subset $X$ of $E$, then the equation (1) has a solution $x \in L^{p}(J, E)$.

Proof. By the theory of scalar linear Volterra integral equations it follows that there exists a nonnegative solution $u(t)$ of the equation

$$
u(t)=a_{1}(t)+b_{1} \int_{D(t)}\|K(t, s)\| a_{2}(s) d s+b_{1} b_{2} \int_{D(t)}\|K(t, s)\| u(s) d s .
$$

More precisely, as the spectral radius $r(\mathcal{K})$ of the Volterra integral operator

$$
\mathcal{K} u(t)=\int_{D(t)}\|K(t, s)\| u(s) d s
$$

is equal to 0 , by Theorem 2.2 from [10] the sequence of successive approximations $u_{n}(t)$ for $(6)$ is convergent; obviously all $u_{n}(t)$ are nonnegative.

Put $B=\left\{x \in L^{p}(D, E):\|x(t)\| \leqslant u(t)\right.$ for a.e. $\left.t \in D\right\}$. Define $F: B \mapsto$ $L^{p}(D, E)$ by

$$
(F x)(t)=\phi\left(t, \int_{D(t)} K(t, s) g(s, x(s)) d s\right) \text { for } x \in B \text { and } t \in D .
$$


Since

$$
\begin{aligned}
\|(F x)(t)\| & =\|\phi(t, S G x(t))\| \leqslant a_{1}(t)+b_{1}\|S G x(t)\| \\
& \leqslant a_{1}(t)+b_{1}\left\|\int_{D(t)} K(t, s) g(s, x(s)) d s\right\| \\
& \leqslant a_{1}(t)+b_{1} \int_{D(t)}\|K(t, s)\|\left(a_{2}(s)+b_{2}\|x(s)\|\right) d s \\
& \leqslant a_{1}(t)+b_{1} \int_{D(t)}\|K(t, s)\| a_{2}(s) d s+b_{1} b_{2} \int_{D(t)}\|K(t, s)\| u(s) d s=u(t)
\end{aligned}
$$

for $x \in B$ and $t \in D$, Lemmas 1 and 2 prove that $F$ is a continuous mapping $B \mapsto B$.

Without loss of generality we shall always assume that all functions from $L^{p}(D, E)$ are extended to $R^{n}$ by putting $x(t)=0$ outside $D$. Moreover, by $1^{\circ}$ (ii) we obtain

$$
\|F(x)(t+h)-F(x)(t)\| \leqslant d(t, h) \text { for } x \in B, t \in D \text { and small }|h|,
$$

where

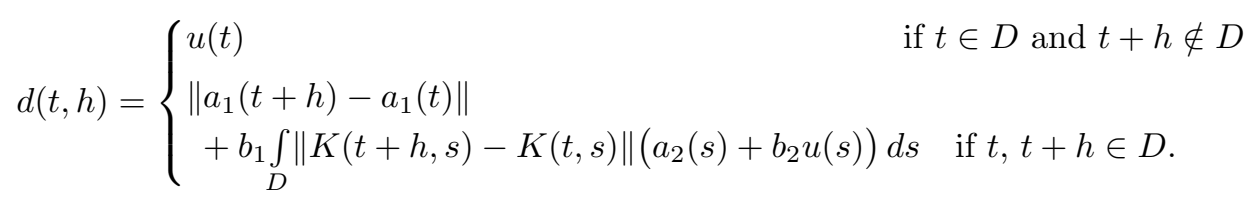

From (2) it follows that for each $z \in L^{1}(D, R)$ we have

$$
\iint_{D \times D} \frac{|z(s)|}{|t-s|^{r}} d s d t=\iint_{D}\left(\int_{D} \frac{d t}{|t-s|^{r}}\right)|z(s)| d s \leqslant Q \int_{D}|z(s)| d s .
$$

In view of (7) the function $(t, s) \mapsto W(t, s)=K(t, s)\left(a_{2}(s)+b_{2} u(s)\right)$ is integrable on $D \times D$. Therefore

$$
\begin{aligned}
\lim _{h \rightarrow 0} \int_{D} d(t, h) d t & =\lim _{h \rightarrow 0} \int_{D}\left(\int_{D}\|K(t+h, s)-K(t, s)\|\left(a_{2}(s)+b_{2} u(s)\right) d s\right) d t \\
& =\lim _{h \rightarrow 0} \int_{D} \int_{D}\|W(t+h, s)-W(t, s)\| d s d t=0
\end{aligned}
$$

for $t \in D$. Hence

$$
\lim _{h \rightarrow 0} \sup _{x \in B} \int_{D(t)}\|(F x)(t+h)-(F x)(t)\| d t=0 .
$$

Next, let $V$ be a countable subset of $B$ such that

$$
V \subset \overline{\operatorname{conv}}(F(V) \cup\{0\}) .
$$


Then $V(t) \subset \overline{\operatorname{conv}}(F(V)(t) \cup\{0\})$ for a.e. $t \in D$, so that

$$
\alpha(V(t)) \leqslant \alpha(F(V)(t)) \text { for a.e. } t \in D \text {. }
$$

Put $v(t)=\alpha(V(t))$ for $t \in D$. From (8) and (9) we deduce that

$$
\lim _{h \rightarrow 0} \sup _{x \in V} \int_{D}\|x(t+h)-x(t)\| d t=0 .
$$

Moreover, $\|x(t)\| \leqslant u(t)$ for all $x \in V$ and a.e. $t \in D$. Consequently, by Lemma 3 , $v \in L^{p}(D, R)$ and

$$
\alpha_{1}(V) \leqslant 2 \int_{D} v(t) d t
$$

According to $1^{\circ}(\mathrm{ii})$, we have $\|\phi(t, x)-\phi(t, y)\| \leqslant b_{1}\|x-y\|$ for $t \in D$ and $x, y \in E$. Then $\alpha(\phi(t, X)) \leqslant b_{1} \alpha(X)$ for any bounded subset $X$ of $E$.

From (7) it is clear that

$$
\int_{D} \frac{a_{2}(s)+b_{2} u(s)}{|t-s|^{r}} d s<\infty \text { for a.e. } t \in D .
$$

Fix $t \in D$ such that the integral (12) is finite. Next, we have

$$
\|K(t, s) g(s, x(s))\| \leqslant a \frac{a_{2}(s)+b_{2} u(s)}{|t-s|^{r}} \text { for } x \in B \text { and } s \in D \text {. }
$$

Case 1. Suppose that the assumptions of Theorem 1 hold. Thus, by (10), (3) and Lemma 3, we get

$$
\begin{aligned}
\alpha(V(t)) & \leqslant \alpha((F V)(t))=\alpha(\phi(t, S G V(t))) \\
& \leqslant b_{1} \alpha\left(\left\{\int_{D(t)} K(t, s) g(s, x(s)) d s: x \in V\right\}\right) \\
& \leqslant 2 b_{1} \int_{D(t)} \alpha(\{K(t, s) g(s, x(s)) d s: x \in V\}) d s \\
& \leqslant 2 b_{1} \int_{D(t)}\|K(t, s)\| \alpha\left(g(s, V(s)) d s \leqslant 2 b_{1} \int_{D(t)}\|K(t, s)\| H(s) \alpha(V(s)) d s\right.
\end{aligned}
$$

i.e.

$$
v(t) \leqslant 2 b_{1} \int_{D(t)}\|K(t, s)\| H(s) v(s) d s
$$

Putting

$$
w(t)=2 b_{1} c \int_{D(t)} \frac{v(s)}{|t-s|^{r}} d s,
$$

where $c=\sup \{\|A(t, s)\| H(s): t, s \in D\}$, we see that $w(t)$ is a continuous function such that $v(t) \leqslant w(t)$ for $t \in D$. Hence 


$$
w(t) \leqslant 2 b_{1} c \int_{D(t)} \frac{w(s)}{|t-s|^{r}} d s .
$$

Arguing similarly as in [8; p. 134-135] we can prove that $w(t)=0$ for $t \in D$. Since $v(t) \leqslant w(t)$, we have $v(t)=0$ for $t \in D$.

Case 2. Suppose that the assumptions of Theorem 2 hold. Thus, by (10), (5) and Lemma 3, we get

$$
\begin{aligned}
\alpha(V(t)) & \leqslant \alpha((F V)(t))=\alpha(\phi(t, S G V(t))) \\
& \leqslant b_{1} \alpha\left(\left\{\int_{0}^{t} K(t, s) g(s, x(s)) d s: x \in V\right\}\right) \\
& \leqslant 2 b_{1} \int_{0}^{t} \alpha(\{K(t, s) g(s, x(s)) d s: x \in V\}) d s \\
& \leqslant 2 b_{1} \int_{0}^{t}\|K(t, s)\| \alpha(g(s, V(s))) d s \leqslant 2 b_{1} \int_{0}^{t}\|K(t, s)\| \omega(\alpha(V(s))) d s,
\end{aligned}
$$

i.e.

$$
v(t) \leqslant 2 b_{1} a \int_{0}^{t} \frac{\omega(v(s))}{(t-s)^{r}} d s \text { for } t \in J .
$$

Putting

$$
w(t)=2 b_{1} a \int_{0}^{t} \frac{\omega(v(s))}{(t-s)^{r}} d s \quad \text { for } t \in J
$$

we see that $w$ is a continuous function such that $v(t) \leqslant w(t)$ for $t \in J$. Hence

$$
w(t) \leqslant 2 b_{1} a \int_{0}^{t} \frac{\omega(w(s))}{(t-s)^{r}} d s \quad \text { for } t \in J .
$$

By the Mydlarczyk theorem [12, Th. 3.1] and assumption (4), the integral equation

$$
z(t)=2 b_{1} a \int_{0}^{t} \frac{\omega(z(s))}{(t-s)^{r}} d s \quad \text { for } \in J
$$

has the unique continuous solution $z(t) \equiv 0$. Applying now theorem on integral inequalities [1, Th. 2], from (14) we deduce that $w(t) \equiv 0$. Thus $v(t)=0$ for $t \in J$.

In view of $(11)$ this shows that $\alpha_{1}(V)=0$, so that $V$ is relatively compact in $L^{1}(D, E)$. On the other hand, the set $B$ has equiabsolutely continuous norms in $L^{p}(D, E)$ and $V \subset B$. Consequently, $V$ is relatively compact in $L^{p}(D, E)$.

Applying now the following Mönch fixed point theorem [11]: 
THEOREM 3. Let $B$ be a closed, convex, and bounded subset of a Banach space such that $0 \in B$. If $F: B \mapsto B$ is a continuous mapping such that for each countable subset $V$ of $B$ the following implication holds

$$
V \subset \overline{\operatorname{conv}}(F(V) \cup 0) \Longrightarrow V \text { is relatively compact, }
$$

then $F$ has a fixed point.

we conclude that there exists $x \in B$ such that $x=F(x)$. Obviously $x$ is a solution of (1).

\section{References}

[1] N. V. Azbieliev, Z. B. Caliuk, Ob integralnych nieravienstvach, Matem. Sbornik 56 (1962), $325-342$.

[2] J. Banaś, K. Goebel, Measure of noncompactness in Banach spaces, Marcel Dekker, New York - Basel, 1980.

[3] J. Banaś, Z. Knap, Integrable solutions of a functional-integral equation, Rev. Mat. Univ. Complutense Madr. 2(1) (1989), 31-38.

[4] G. Emmanuele, Existence of solutions of a functional-integral equation in infinite dimensional Banach spaces, Czech. Math. J. 44(4) (1994), 603-609.

[5] M. A. Darwish, Weakly singular functional-integral equation in infinite dimensional Banach spaces, Appl. Math. Comp. 136 (2003), 123-129.

[6] G. Gripenberg, Unique solutions of some Volterra integral equations, Math. Scand. 48 (1981), $59-67$.

[7] H. P. Heinz, On the behaviour of measures of noncompactness with respect to differentiation and integration of vector-valued functions, Nonlinear Anal. 7 (1983), 1351-1371.

[8] J. Januszewski, On Volterra integral equations with weakly sigular kernel in Banach spaces, Demonstr. Math. 26 (1993), 131-136.

[9] W. Kołodziej, Wybrane rozdziaty analizy matematycznej, PWN, Warszawa 1982.

[10] M. A. Krasnoselskii, Približennoe resenie operatornyh uravnenij, Nauka, Moskva 1969.

[11] H. Mönch, Boundary value problems for nonlinear ordinary differential equations of second order in Banach spaces, Nonlinear Anal. 4 (1980), 985-999.

[12] W. Mydlarczyk, The existence of nontrivial solutions of Volterra equations, Math. Scand. 68 (1991), 83-88.

[13] D. O'Regan, M. Meehan, Existence Theory for Nonlinear Integral and Integrodifferential Equations, Kluwer, Dordrecht, 1998.

[14] B. Sadovskii, Limit-compact and condensing mappings, Russian Math. Surveys 27 (1972), $85-155$.

[15] S. Szufla, On the Hammerstein integral equation with weakly singular kernel, Funkcialaj Ekvacioj 34 (2) (1991), 279-285.

[16] S. Szufla, Appendix to the paper "An existence theorem for the Urysohn integral equation in Banach spaces", Comm. Math. Univ. Carolinae 25 (1984), 763-764.

[17] S. Szufla, On the Volterra integral equation with weakly singular kernel, Mathem. Bohem. 131(3) (2006), 225-231.

Faculty of Mathematics and Computer Science

Adam Mickiewicz University

Umultowska 87

61-614 Poznań

Poland

szukala@amu.edu.pl
(Received 3001 2007)

(Revised 0809 2008) 